\title{
The foot-related clinical characteristics of people with diabetes in an Australian regional setting
}

\author{
Byron Perrin ${ }^{1,2,3^{*}}$, Marcus Gardner ${ }^{3}$, Susan Kennett ${ }^{4}$ \\ From Australasian Podiatry Council Conference 2011 \\ Melbourne, Australia. 26-29 April 2011
}

\section{Background}

The podiatrists of the regional Victorian health organisations Bendigo Health (BH) and Bendigo Community Health Services (BCHS) recently evaluated a collaborative podiatric model of care. This paper describes the clinical and demographic characteristics of the patients seen within this model over a three-month period.

\section{Methods}

A three-month prospective clinical audit of the podiatry services involved in the podiatric model of care was undertaken. Basic demographic variables were recorded in addition to the UT risk classification at baseline and incidence of ulceration. Statistical analysis was undertaken to explore the differences in the demographic variables with respect to both risk category at baseline and incidence of ulceration.

\section{Results}

576 patients were seen during the three-month period. Mean age was $71.3 \pm 11.6$ years, with males accounting for $53.3 \%$ of the cohort. The majority (95.8\%) of the sample had type 2 diabetes and the mean duration of diabetes was $12.08 \pm 10.0$ years. At baseline, $51.4 \%$ of the cohort was classified as having "no neuropathy" and $10.6 \%$ classified as having an "active pathology". Those at higher risk of future foot pathology at baseline were younger $(\mathrm{F}=11.9, \mathrm{p}<0.0005)$ and had a longer duration of diabetes $(\mathrm{F}=31.7, \mathrm{p}<0.0005)$. Males duration of diabetes $(t=-3.3, p=0.002)$. The proportion of people with type 1 diabetes who developed incident ulceration was higher than for people with type 2 diabetes $\left(\chi^{2}=9.1, \mathrm{p}=0.003\right)$.

\section{Conclusions}

The foot health of this large cohort ranges across the entire spectrum of diabetes-related foot complications. There are consistencies with other relevant populations with respect to age, gender, diabetes type and the proportion of males and adults with type 1 diabetes at higher risk of future foot pathology. Although it is unusual to see those at higher risk of problems being a younger age, this may be offset by a longer duration of diabetes. The three-month incidence of ulceration was high, reflecting the clinical nature of the cohort.

\section{Author details \\ 'La Trobe Rural Health School, La Trobe University, Bendigo, Victoria, 3550, Australia. ${ }^{2}$ Musculoskeletal Research Unit, La Trobe University, Bundoora, Victoria, 3086, Australia. ${ }^{3}$ Bendigo Health, Bendigo, Victoria, 3550, Australia. ${ }^{4}$ Bendigo Community Health Services, Bendigo, Victoria, 3550, Australia.}

Published: 20 May 2011

doi:10.1186/1757-1146-4-S1-P44

Cite this article as: Perrin et al:: The foot-related clinical characteristics of people with diabetes in an Australian regional setting. Journal of Foot and Ankle Research 2011 4(Suppl 1):P44. Thirty-six $(6.3 \%)$ people developed incident diabetesrelated foot ulceration. Those that developed ulceration were younger $(t=3.5, \mathrm{p}=0.001)$ and had a longer

\footnotetext{
* Correspondence: b.perrin@latrobe.edu.au

'La Trobe Rural Health School, La Trobe University, Bendigo, Victoria, 3550, Australia

Full list of author information is available at the end of the article
}

(c) 2011 Perrin et al; licensee BioMed Central Ltd. This is an open access article distributed under the terms of the Creative Commons 